\title{
Mid-infrared imaging of the young binary star Hen 3-600: Evidence for a dust disk around the primary
}

\author{
Ray Jayawardhana ${ }^{1,3}$, Lee Hartmann ${ }^{1}$, Giovanni Fazio ${ }^{1}$, \\ R. Scott Fisher ${ }^{2,3}$, Charles M. Telesco ${ }^{2,3}$ and Robert K. Piña ${ }^{2}$
}

\begin{abstract}
We present high-resolution mid-infrared observations of the nearby late-type young binary system Hen 3-600. The binary, at a distance of $\sim 50 \mathrm{pc}$, could be a member of the TW Hydrae Association, the nearest known group of young stars, with an age of a few million years. Our images make it possible for the first time to determine which star in the pair, separated by 1.4", harbors the mid-infrared excess detected by IRAS. In the near-infrared, where the radiation is primarily photospheric, Hen 3-600A (M3) and Hen 3-600B (M3.5) have a flux ratio of 1.6. At $4.8 \mu \mathrm{m}, 10.8 \mu \mathrm{m}$, and $18.2 \mu \mathrm{m}$, the primary becomes increasingly dominant over the secondary, suggesting that most of the circumstellar dust in the system resides around Hen 3-600A. Comparison of the spectral energy distribution (SED) of Hen 3-600A to the median SED of classical T Tauri stars suggests that its disk may be truncated by the secondary and provides tentative evidence for a central disk hole. The distribution of dust in the Hen 3-600 system may provide important clues to the formation and evolution of protoplanetary disks in close binaries.
\end{abstract}

Subject headings: Accretion, accretion disks - Stars: Circumstellar Matter - Stars: Binaries - Stars: Formation - Stars: Pre-Main Sequence

\section{Introduction}

Over the past decade, infrared and millimeter observations have provided strong support for the single star plus accretion disk model of star formation (Shu, Adams, \& Lizano 1987; also

\footnotetext{
${ }^{1}$ Harvard-Smithsonian Center for Astrophysics, 60 Garden St., Cambridge, MA 02138; Electronic mail: rayjay@cfa.harvard.edu

${ }^{2}$ Department of Astronomy, University of Florida, Gainesville, FL 32611

${ }^{3}$ Visiting Astronomer, Cerro Tololo Interamerican Observatory, National Optical Astronomy Observatories, which is operated by the Association of Universities for Research in Astronomy, Inc. (AURA) under cooperative agreement with the National Science Foundation.
} 
see Hartmann 1998). The disk contains most of the system's angular momentum and may help accrete the bulk of the central star's mass. It is also the likely site of planet formation.

This scenario must be more complicated in most systems because a majority of young, low-mass stars are members of binary systems (Ghez et al. 1993, 1997; Leinert et al. 1993; Reipurth \& Zinnecker 1993; Simon et al. 1995). A young binary system may contain up to three distinct disks: circumstellar disks around one or both stars, with disk radii smaller than the binary's periastron separation, and a circumbinary disk lying entirely outside the binary orbit (cf. Jensen \& Mathieu 1997). These disks are remnants of star birth and thus may provide clues to binary formation mechanisms. Furthermore, the stars and the disk(s) in a binary influence each component's evolution through dynamical interactions (e.g., Lin \& Papaloizou 1993; Artymowicz \& Lubow 1994, 1996). Therefore, the study of disks in binary systems is crucial to our understanding of both star and planet formation processes.

Here we report high-resolution mid-infrared imaging observations of the nearby, young,

late-type binary Hen 3-600. It was first identified by de la Reza et al. (1989) as a T Tauri pair in the vicinity of another isolated young star TW Hydrae based on the presence of $\mathrm{H} \alpha$ emission and the Li I $6708 \AA$ absorption line. Its coincidence with the IRAS source 1108-3715 was interpreted as being due to thermal dust emission associated with one or both stars in the binary. More recently, several groups have suggested that Hen 3-600, TW Hya and a number of other active, young stars in the same region of the sky form a physical association with an age of $10 \mathrm{Myr}$ (Gregorio-Hetem et al. 1992; Kastner et al. 1997; Soderblom et al. 1998; Webb et al. 1999; see Jensen, Cohen, \& Neuhäuser 1998 for a different viewpoint). At a distance of 50 pc, the "TW Hydrae Association" is the nearest known group of young stars, and is ideally suited to studies of circumstellar disk evolution.

The primary goal of our observations was to determine whether the thermal dust emission in the Hen 3-600 system originates from one or both stars in the binary. A secondary motivation was to place useful constraints on the nature of the dust disk(s) by comparing the near- to far-infrared spectral energy distribution (SED) with observations of T Tauri disks in Taurus.

\section{Observations and Results}

Hen 3-600 was observed on February 23, 1999 with the 4-m Blanco telescope at Cerro Tololo Interamerican Observatory using OSCIR, the University of Florida mid-infrared camera. OSCIR uses a $128 \times 128 \mathrm{Si}$ :As Blocked Impurity Band (BIB) detector developed by Boeing. On the CTIO 4 -m telescope, OSCIR has a plate scale of 0.183 " / pixel, which gives a field of view of 23 " $\times 23$ ". Our observations were made using the standard chop/nod technique with a chopper throw of 23 " in declination. Images of Hen 3-600 were obtained in the $\mathrm{K}(2.2 \mu \mathrm{m}), \mathrm{M}(4.8 \mu \mathrm{m}), \mathrm{N}(10.8 \mu \mathrm{m})$, and IHW18 $(18.2 \mu \mathrm{m})$ bands, and flux calibrated using the standard stars $\lambda$ Vel and $\gamma$ Cru. On-source 
integration times for Hen 3-600 were 300 seconds in K, 300 seconds in M, 600 seconds in N, and 600 seconds in IHW18. Additional information on OSCIR is available on the World-Wide Web at www.astro.ufl.edu/iag/. Our final stacked images at each of the four wavelengths are shown in Figure 1.

In the $\mathrm{K}$ band, the two components of the binary are clearly resolved, with a peak-to-peak separation of $1.4^{\prime \prime}$ and a position angle of $215^{\circ} \pm 3^{\circ}$, consistent with previous observations. The flux ratio we measure for the $\mathrm{A}$ and $\mathrm{B}$ components, $F_{A} / F_{B}=1.6$, agrees extremely well with that from Webb et al.'s (1999) K magnitudes based on IRTF photometry. In the M-band, the two stars are again well resolved, with a flux ratio of $\sim 1.9$. In the $\mathrm{N}$-band, only the primary $(\mathrm{A})$ is clearly visible. Our flux measurement of $900 \pm 90 \mathrm{mJy}$ is consistent with the IRAS $12 \mu \mathrm{m}$ flux of $990 \mathrm{mJy}$. By subtracting an appropriately scaled PSF star from the position of Hen 3-600A, we are able to place an upper limit of $50 \mathrm{mJy}$ on the flux from B at N. In the IHW18 band, again only A appears on our image, with a total flux of $1500 \pm 150 \mathrm{mJy}$, which is consistent with the IRAS $25 \mu \mathrm{m}$ measurement of $1750 \mathrm{mJy}$. (The errors are primarily due to uncertainties in flux calibration.)

\section{Discussion}

\subsection{Location and nature of circumstellar dust}

Our mid-infrared images show that the IRAS-detected excess emission originates from Hen 3-600A, and not from Hen 3-600B, implying that the primary has a circumstellar disk while the secondary does not. From Keck LRIS spectra, Webb et al. (1999) measure H $\alpha$ equivalent widths of $-21.8 \AA$ and $-7.14 \AA$ for $\mathrm{A}$ and $\mathrm{B}$ respectively. Taken together, our mid-infrared images and

Webb et al.'s spectra suggest that A is a classical T Tauri star (CTTS) with an actively accreting disk, while B is a weak-line T Tauri star (WTTS) without an optically thick inner disk.

In a spectroscopic and photometric study of 12 pre-main-sequence close binary systems, Prato \& Simon (1997) found that in every case both components exhibited CTTS characteristics. Thus, they suggested that the transition of disk optical depth, from $\tau>1$ to $\tau<1$, is roughly coeval for both stars in a close binary and that a circumbinary envelope could regulate the common evolution of the inner disks. Our detection of a circumstellar disk around only the primary, and not the secondary, in Hen 3-600 is inconsistent with that scenario. However, it does agree with Prato (1998) who finds evidence for only circumprimary disks in 4 close binaries in a larger sample of 25 systems. We further note that in a study of 39 wide pre-main sequence binary systems, Hartigan, Strom, \& Strom (1994) found that 6 systems were composed of CTTS/WTTS pairs, as judged from the $\mathrm{H} \alpha$ emission (see Figure 8 of Hartigan et al. 1994). The last step in the evolution from the CTTS phase to the WTTS state may take place on relatively short timescales. It is 
possible that Hen 3-600 is a transition system with one component already evolved into a WTTS while the other is still in the process of doing so.

We plot the composite spectral energy distribution (SED) of Hen 3-600A in Figure 2 and compare it to the median SED for a classical T Tauri star of similar spectral type and to the SED of the HD 98800 quadruple system. The solid line on the plot corresponds to the median CTTS SED for stars in Taurus, normalized at H (D'Alessio et al. 1999); the dashed lines show the quartile fluxes to provide some idea of the range of observed fluxes. The dip at $4.8 \mu \mathrm{m}$ (M filter) in the spectrum of Hen 3-600A is suggestive of a possible central hole in the disk. However, this result needs to be confirmed with higher-precision photometry at wavelengths between $2 \mu \mathrm{m}-10 \mu \mathrm{m}$. At $60 \mu \mathrm{m}$ and $100 \mu \mathrm{m}$, the emission from Hen 3-600A falls well below that expected for a median 100 AU CTTS disk, implying that the disk may be significantly smaller in radius. Assuming an optically thick disk with a temperature distribution $T \propto r^{-1 / 2}$ (Beckwith et al. 1990) suggests that truncating the disk at an outer radius of $\sim 25 \mathrm{AU}$ would help account for the reduction in long-wavelength fluxes. Similar dips at $5-10 \mu \mathrm{m}$ and again at far-infrared wavelengths are also seen in HD 98800, where the dust is apparently associated with one of the two binary pairs (Gehrz et al. 1999).

Theoretical calculations suggest that circumstellar disks will be truncated by the tidal effects of a companion star in circular orbit at approximately 0.9 of the average Roche lobe radius (Artymowicz \& Lubow 1994), which for roughly equal mass stars in the Hen 3-600 system would be at about a third of the orbital radius (Papaloizou \& Pringle 1977). Given a binary separation of $1.4 "$, the disk of the primary should then be confined to $<0.5$ " angular radius. It is interesting to note that in another young close binary, L1551 IRS5 in Taurus, compact disks have been observed around each star with the Very Large Array (VLA) at $7 \mathrm{~mm}$ (Rodriguez et al. 1998). In that case, the disk radii appear to be about a quarter of the binary separation distance. (Of course, it is possible that these outer radii are the result of instrument sensitivity limits rather than disk truncation.)

\subsection{Age and evolutionary status of Hen 3-600}

Hen 3-600 appears to be one of several nearby young stellar systems in the vicinity of TW Hydrae. Based on strong X-ray fluxes from all five systems, Kastner et al. (1997) concluded that the group forms a physical association at a distance of $\sim 50 \mathrm{pc}$ with an age of $20 \pm 10 \mathrm{Myr}$. Webb et al. (1999) have identified five more T Tauri star systems in the same region of the sky as candidate members of the "TW Hya Association" (TWA), based on the same signatures of youth - namely high X-ray flux, large Li abundance, and strong chromospheric activity- and the same proper motion as the original five members. Furthermore, they suggest that the wide binary HR 4796 is also part of the Association, even though its Hipparcos parallactic distance of $67 \mathrm{pc}$ 
places it further away than most other members of the group. The three other TWA stars with Hipparcos distances -TW Hya, HD 98800, and TWA 9- are at 56, 47 and 50 pc, respectively.

If all proposed members of the TWA do indeed form a coeval group with a common origin, they would provide an excellent sample for studying disk evolution timescales. In particular, using the Hipparcos distance, and the D'Antona \& Mazzitelli (1994) evolutionary tracks, it is possible to estimate an age of $8 \pm 3 \mathrm{Myr}$ for the M2.5 star HR 4796B (Jayawardhana et al. 1998). This age is consistent with the upper bound provided by the measurement of the strong Li absorption line at $6708 \AA$ (Stauffer et al. 1995); for this mass range, Li is predicted to be rapidly destroyed in the stellar interior at ages $\sim 9-11 \mathrm{Myr}$ (see, e.g., D'Antona \& Mazzitelli 1994). We note that HR 4796B and Hen 3-600A have comparable Li equivalent widths and $L_{X} / L_{b o l}$ ratios. Based on similar considerations, Soderblom et al. (1998) derive an age of 7-12 Myr for the HD 98800 quadruple system. A lower limit to the age of a few Myr is indicated by the isolated location of HR 4796, HD 98800, Hen 3-600, and the other TWA stars, since most stars of comparable or smaller ages are found in regions of molecular clouds and substantial interstellar dust extinction (Leisawitz, Bash, \& Thaddeus 1989).

A spatially-resolved circumstellar disk was recently imaged around the A star HR 4796A at mid-infrared wavelengths (Jayawardhana et al. 1998; Koerner et al. 1998; Telesco et al. 1999). The presence of an inner disk hole in HR 4796A, first inferred by Jura et al. (1993) on the basis of the SED and now confirmed by mid-infrared imaging, may provide evidence for coagulation of dust into larger bodies on a timescale similar to that suggested for planet formation in the solar system (e.g., Strom et al. 1989; Strom, Edwards, \& Skrutskie 1993; Podosek \& Cassen 1994). If TWA stars are indeed coeval, it would be of significant interest to determine whether similar disk evolution has ocurred in other systems.

However, given that the apparent proper motions of the TWA stars are primarily due to solar reflex motion, it is difficult to establish a common origin for the candidate group members (Jensen, Cohen, \& Neuhäuser 1998; Soderblom et al. 1998; Hoff, Henning \& Pfau 1998). In particular, without a Hipparcos distance measurement, the position of Hen 3-600 on the H-R diagram is highly uncertain. At an assumed distance of 50 pc, Webb et al. (1999) obtain an age of a few Myr for Hen 3-600A and B from PMS evolutionary tracks.

In summary, it is likely that Hen 3-600 is younger than $10 \mathrm{Myr}$, based on the presence of $\mathrm{Li}$, and older than $\sim 1 \mathrm{Myr}$, given the lack of an associated parent molecular cloud. Possible membership of the TWA suggests an age close to the upper end of this range while its CTTS characteristics point to the lower end. A more accurate age estimate may not be possible until its distance is directly measured or a common origin for the TWA stars is firmly established.

\subsection{Implications for disk formation and evolution in binaries}


Nearly half of the known pre-main-sequence stars in Taurus are WTTS, i.e., they have $\mathrm{H} \alpha$ equivalent widths $<10 \AA$ and no infrared excess, implying a lack of accretion disks. It has been argued that many, if not most, WTTS have binary stellar companions which render disks dynamically unstable (Jensen, Mathieu, \& Fuller 1994, 1996; Osterloh \& Beckwith 1995). Therefore, "mixed" binary systems like Hen 3-600, where one star is a CTTS and the other a WTTS, present somewhat of a puzzle.

The exact configuration of disks - circumprimary, circumsecondary and/or circumbinary-in a binary system may depend on the details of its formation. Smoothed particle hydrodynamics (SPH) simulations by Bate \& Bonnell (1997) show that the fractions of infalling material that are captured by each protostar and the fraction which forms a circumbinary disk depend on the binary's mass ratio and the parent cloud's specific angular momentum, $j_{\text {infall }}$. For accretion with low $j_{\text {infall }}$, most of the infalling material is captured by the primary. For gas with intermediate $j_{\text {infall }}$, the fraction captured by the primary decreases and that captured by the secondary increases. For higher $j_{\text {infall }}$, more and more gas goes into a circumbinary disk instead of circumstellar disks. Thus, it could be that infall from a low $j_{\text {infall }}$ cloud led to a more massive disk around the primary in Hen 3-600. However, given their roughly equal masses, it is not clear why one star would capture much more material than the other. One possibility is that as protostars, the two components had very different masses, with what is now the primary accumulating most of its mass at the end of accretion. Another possibility is that there is a very low-mass, so far undetected, close companion around Hen 3-600B which accelerated the depletion of its disk.

It is likely that there is not a universal evolutionary timescale for protoplanetary disks, especially when the influence of companion stars is taken into account. Detailed studies of disks around a large sample of young binaries, including other candidate members of the TWA, may provide new insights to resolve this puzzle.

We wish to thank the staff of CTIO for their outstanding support. We are also grateful to Jim DeBuizer for his assistance at the telescope. The research at CfA was supported by NASA grant NAG5-4282 and the Smithsonian Institution. The research at the University of Florida was supported by NASA, NSF, and the University of Florida. 


\section{REFERENCES}

Artymowicz, P. \& Lubow, S.H. 1994, ApJ, 421, 651

Artymowicz, P. \& Lubow, S.H. 1996, ApJ, 467, 77

Bate, M.R. \& Bonnell, I.A. 1997, MNRAS, 285, 33

Beckwith, S.V.W., Sargent, A.I., Chini, R.S., \& Guesten, R. 1990, AJ, 99, 924

D’Alessio, P., Calvet, N., Hartmann, L., Lizano, S., \& Cantó, J. 1999, ApJ, submitted

D’Antona , F., \& Mazzitelli, I. 1994, ApJ Suppl. Ser. 90, 467

de la Reza, R., et al. 1989, ApJL, 343, 61

Gehrz, R.D., Smith, N., Low, F.J., Krautter, J., Nollenberg, J.G., \& Jones, T.J. 1999, ApJL, 512, L55

Ghez, A.M., Neugebauer, G., \& Matthews, K. 1993, AJ, 106, 2005

Ghez, A.M., McCarthy, D.W., Patience, J.L., \& Beck, T.L. 1997, ApJ, 481, 378

Gregorio-Hetem, J., et al. 1992, AJ, 103, 549

Hartigan, P., Strom, K.M., \& Strom, S.E. 1994, ApJ, 427, 961

Hartmann, L. 1998, Accretion Processes in Star Formation, Cambridge University Press

Hoff, W., Henning, T., \& Pfau, W. 1998, A\&A, 336, 242

Jayawardhana, R., Fisher, S., Hartmann, L., Telesco, C., Piña, R. \& Fazio, G. 1998, ApJ, 503, L79

Jensen, E.L.N., Mathieu, R.D., \& Fuller, G.A. 1994, ApJ, 429, 29

Jensen, E.L.N., Mathieu, R.D., \& Fuller, G.A. 1996, ApJ, 458, 312

Jensen, E.L.N. \& Mathieu, R.D. 1997, AJ, 114, 301

Jensen, E.L.N., Cohen, D.H., \& Neuhäuser, R. 1998, AJ, 116, 414

Jura, M., Zuckerman, B., Becklin, E.E., \& Smith, R.C. 1993, ApJL, 418, 37

Kastner, J.H., Zuckerman, B., Weintraub, D.A. \& Forveille, T. 1997, Science, 277, 67

Koerner, D., Werner, M., Ressler, M., \& Backman, D. 1998, ApJL, 503, L83

Leinert, Ch., et al. 1993, A\&A, 278, 129

Leisawitz, D., Bash, F. \& Thaddeus, P. 1989, ApJ Suppl. Ser. 70, 731

Lin, D.N.C. \& Papaloizou, J.C.B. 1993, in Protostars and Planets III (eds. Levy, E.H. \& Lunine, J.I.) (Tucson: Univ. Arizona Press), 749

Osterloh, M. \& Beckwith, S.V.W. 1995, ApJ, 439, 288

Papaloizou, J., \& Pringle, J.E. 1977, MNRAS, 181, 441

Podosek, F.A., \& Cassen, P. 1994 , Meteoritics, 29, 6 
Prato, L. \& Simon, M. 1997, ApJ, 474, 455

Prato, L. 1998, BAAS, 193, 8904

Reipurth, B. \& Zinnecker, H. 1993, A\&A, 278, 81

Rodriguez, L.F., et al. 1998, Nature, 395, 355

Shu, F.H., Adams, F.C., \& Lizano, S. 1987, Ann. Rev. A\&A, 25, 23

Simon, M., et al. 1995, ApJ, 443, 625

Soderblom, D.R., et al. 1998, ApJ, 498, 385

Stauffer, J.R., Hartmann, L.W. \& Barrado y Navascues, D. 1995, ApJ, 454, 910

Strom, K.M., Strom, S.E., Edwards, S., Cabrit, S. \& Skrutskie, M.F. 1989, ApJ, 97, 1451

Strom, S.E., Edwards, S., \& Skrutskie, M.F. 1993, in Protostars and Planets III (eds. Levy, E.H. \& Lunine, J.I.) (Tucson: Univ. Arizona Press), 837

Sylvester, R.J., Skinner, C.J., Barlow, M.J., \& Mannings, V. 1996, MNRAS, 279, 915

Telesco, C., et al. 1999, ApJ, submitted

Webb, R.A., Zuckerman, B., Platais, I., Patience, J., White, R.J., Schwartz, M.J., \& McCarthy, C. 1999, ApJ, 512, L63 


\section{Figure Captions}

Figure 1. Final, stacked OSCIR images of Hen 3-600 in K $(2.2 \mu \mathrm{m})$ upper left, M $(4.8 \mu \mathrm{m})$ upper right, N $(10.8 \mu \mathrm{m})$ lower left, and IHW18 $(18.2 \mu \mathrm{m})$ lower right filters, smoothed with a 3-pixel Gaussian. The bars below each panel indicate the intensity scale. The plate scale is 0.183 " / pixel.

Figure 2. (a) Composite spectral energy distribution of Hen 3-600A from near- to far-infraed wavelengths based on measurements of Webb et al. (1999), IRAS, and this paper. (b) The SED of HD 98800, for comparison, based on fluxes reported by Sylvester et al. (1996). The solid line in each panel is the median SED for Taurus CTTS, normalized at H (from D'Alessio et al. 1999), and the dashed lines show the quartile fluxes to provide some idea of the range of observed fluxes. 

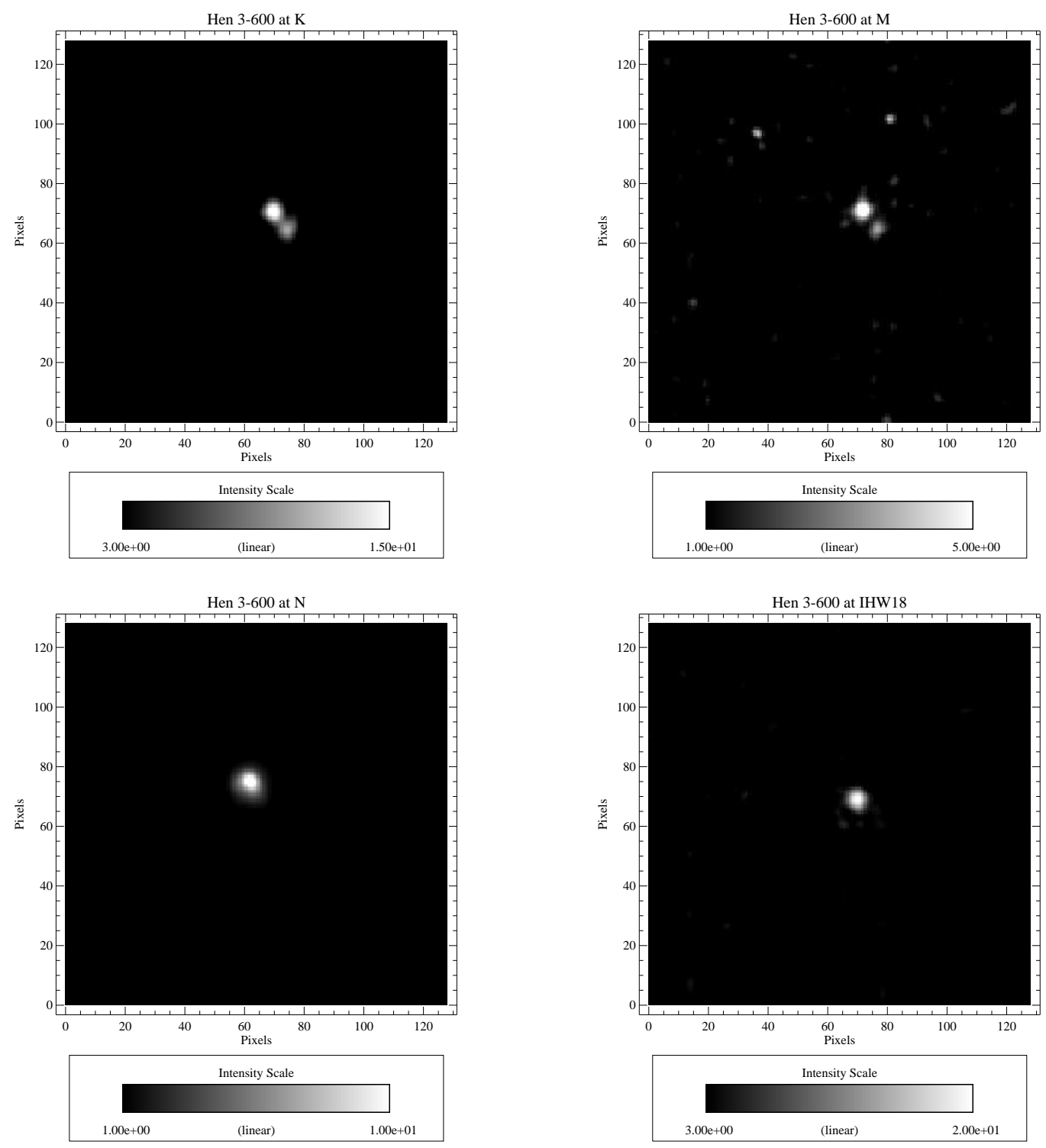

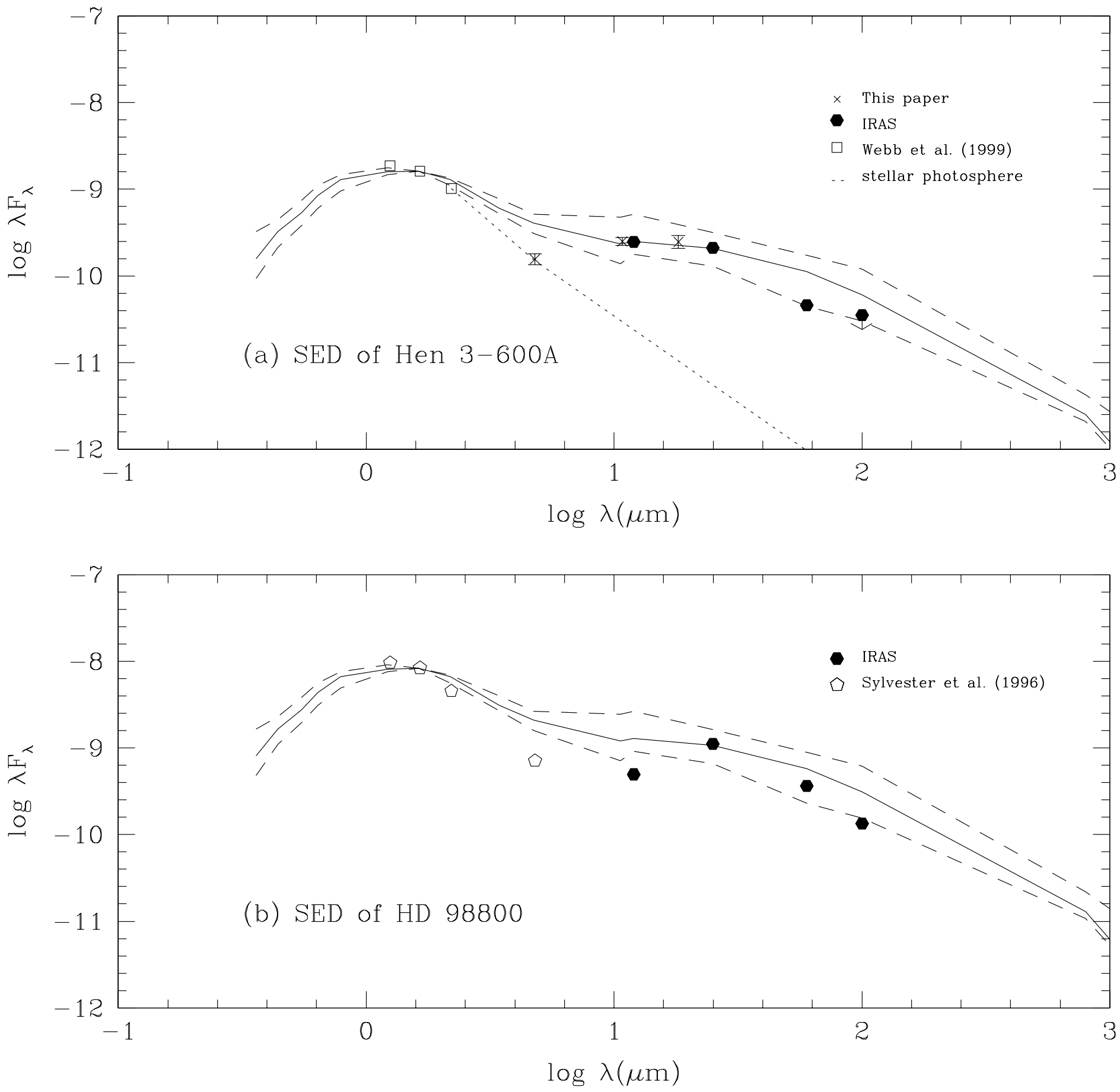